\title{
Calibration and verification of streaked optical pyrometer system used for laser-induced shock experiments
}

\author{
Zhiyu He ${ }^{1}$, Guo Jia ${ }^{1}$, Fan Zhang ${ }^{1}$, Xiuguang Huang ${ }^{1,2}$, Zhiheng Fang ${ }^{1}$, Jiaqing Dong ${ }^{1}$, Hua Shu ${ }^{1}$, \\ Junjian $\mathrm{Ye}^{1}$, Zhiyong Xie ${ }^{1}$, Yuchun $\mathrm{Tu}^{1}$, Qili Zhang ${ }^{3}$, Erfu Guo ${ }^{1}$, Wenbing Pei ${ }^{1,2}$, and Sizu Fu ${ }^{1,2}$ \\ ${ }^{1}$ Shanghai Institute of Laser Plasma, CAEP, Shanghai 201800, China \\ ${ }^{2}$ IFSA Collaborative Innovation Center, Shanghai Jiao Tong University, Shanghai 200240, China \\ ${ }^{3}$ Institute of Applied Physics and Computational Mathematics, Beijing 100094, China \\ (Received 9 January 2019; revised 28 May 2019; accepted 10 June 2019)
}

\begin{abstract}
Although the streaked optical pyrometer (SOP) system has been widely adopted in shock temperature measurements, its reliability has always been of concern. Here, two calibrated Planckian radiators with different color temperatures were used to calibrate and verify the SOP system by comparing the two calibration standards using both multi-channel and single-channel methods. A high-color-temperature standard lamp and a multi-channel filter were specifically designed for the measurement system. To verify the reliability of the SOP system, the relative deviation between the measured data and the standard value of less than $5 \%$ was calibrated out, which demonstrates the reliability of the SOP system. Furthermore, a method to analyze the uncertainty and sensitivity of the SOP system is proposed. A series of laserinduced shock experiments were conducted at the 'Shenguang-II' laser facility to verify the reliability of the SOP system for temperature measurements at tens of thousands of kelvin. The measured temperature of the quartz in our experiments agreed fairly well with previous works, which serves as evidence for the reliability of the SOP system.
\end{abstract}

Keywords: laser-induced shock waves; shock temperature measurement; streaked optical pyrometer

\section{Introduction}

Temperature is one of the most important parameters for characterizing the thermodynamic state of matter. Temperature measurements of materials under extreme conditions play an important role in military applications, inertial confinement fusion $^{[1]}$, high-energy-density physics ${ }^{[2,3]}$, and fundamental material studies ${ }^{[4]}$. For instance, the establishment of the theoretical equation of state (EOS) model at ultra-high pressures is very sensitive to the temperature. Shock temperature measurements are among the important means to examine and test the theoretical EOS model under extreme conditions. High-power lasers have been increasingly used as drivers for shock-wave experiments, and can produce extremely high pressures ${ }^{[5-11]}$.

To date, measurements of the mechanical parameters, such as the shock-wave velocity and pressure, of a system have reached a relatively mature state in shock-wave experiments to study the EOS. However, accurately measuring thermo-

Correspondence to: G. Jia, Shanghai Institute of Laser Plasma, Shanghai 201800, China. Email: 387890448@qq.com dynamic parameters, such as the temperature, remains a difficult task because of the measurement complexity, precision requirements for the instruments, and the establishment of the calculation model ${ }^{[3]}$. To date, the optical radiation method has been widely used for transient measurements of the shock temperature. The shock temperature of materials can be inferred from the recorded self-emission of the shocked materials by comparing the emission to a calibrated Planckian radiator. A streaked optical pyrometer (SOP) is commonly used to measure the self-emission ${ }^{[5,12-16]}$.

Although the SOP system has been widely adopted in shock temperature measurements, the reliability of this system has always been questioned. Firstly, the method of temperature measurement based on Planck's theory has been proposed for decades, but few people have validated the SOP system with more than one standard source to prove its reliability. Secondly, Zeldovich and Raizer ${ }^{[3]}$ and Tan ${ }^{[17]}$ have presented different physical understandings of emissivity. The former held that emissivity is a function of frequency, while the latter considered that emissivity is independent of frequency and the measured surface temperature is the 
composition of the temperature behind the shock front. Thirdly, some researchers have proposed that the problem of surface radiation and absorption is complicated and difficult to explain ${ }^{[18,19]}$. Effective verification and assessment of the reliability of the diagnostic method is a common difficulty. Therefore, it is necessary to develop methods to calibrate and verify the diagnostic method and assess the reliability of the SOP system. This ensures the validity and rationality of the obtained shock temperature data and addresses concerns regarding the feasibility of the SOP system applied to hightemperature measurements.

Here, we present a method to calibrate and verify the SOP system using two kinds of Planckian radiator standard lamp sources with different color temperatures to calibrate the system. The two lamps are regarded as the standard for each other in the SOP system. The relative deviation between the measured data and the standard value was calibrated for, enabling the SOP system to be used at thousands of kelvin. In addition, a method to analyze the uncertainty and sensitivity of the SOP system is described. A series of laserinduced shock experiments were conducted at a laser facility to verify the reliability of the SOP system for temperature measurements at tens of thousands of kelvin.

\section{Verification of the SOP system}

\subsection{Theoretical basis}

The temperature measurement diagnostic system is based on Planck's black-body theory, which states that all bodies of finite temperature emit with a spectral radiance characteristic of their thermal state ${ }^{[12]}$. For standard lamp and shock temperature experiments that can be regarded as a gray body, we relate the temperature $T$ to the spectral radiance $L(\lambda)$ by Planck's law:

$$
L(\lambda)=\frac{\varepsilon(\lambda)}{\lambda^{5}} \frac{2 h c^{2}}{e^{h c / \lambda k T}-1},
$$

where $h$ is Planck's constant, $c$ is the speed of light, $k$ is the Boltzmann constant, $\lambda$ is the wavelength of the SOP channel, and $T$ is the temperature of the body. The standard lamps are specially designed so that the emissivity $\varepsilon$ has a weak correlation with the wavelength in our calibrations.

Two calibrated, Planckian radiator, standard lamps with different color temperatures were used in the calibration and verification of the SOP system, and are regarded as the known standard and unknown source for each other. One of the lamps is a CSTM-USLR-S12F produced by American Labsphere Incorporated, a National Institute of Standards and Technology (NIST)-traceable halogen tungsten lamp $(T \sim 3000 \mathrm{~K})$ of known spectral radiance accurate to $\sim 1 \%$, with an emissivity of $(6 \pm 0.04) \times 10^{-3}$. The uniformity of the lamp emissivity is better than $98 \%$ according to the original

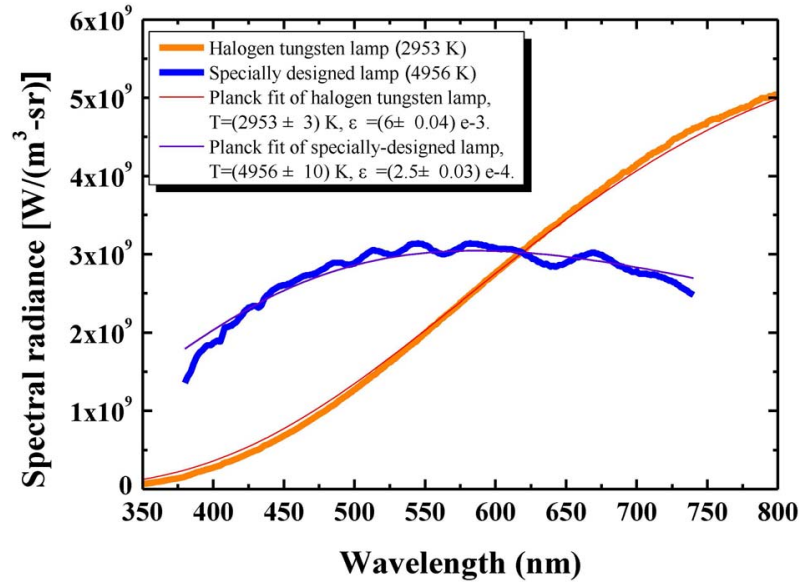

Figure 1. Spectral radiance and Planck fit of the lower-color-temperature $(\sim 3000 \mathrm{~K})$ halogen tungsten lamp (thick orange line and thin red line) and the higher-color-temperature $(\sim 5000 \mathrm{~K})$ specially designed lamp (thick blue line and thin purple line).

calibration report. The other lamp has a customized filter placed in front of the outlet of a standard halogen tungsten lamp (CSTM-USLR-S12F), also a specially designed NISTtraceable Planckian radiator with a higher color temperature $(T \sim 5000 \mathrm{~K})$. The filter completely covers the outlet and is spatially uniform, with a multilayer dielectric film structure to make the lamp consistent with the theoretical Planck curve at $\sim 5000 \mathrm{~K}$ in the visible range (380-740 nm). The measured color temperature and emissivity of this lamp are $(4956 \pm 10) \mathrm{K}$ and $(2.5 \pm 0.03) \times 10^{-4}$, respectively, according to the original test report. The deviation between the measured spectral radiance and the theoretical Planck curve is designed to be lower than $10 \%$ in the visible range. The spectral radiance and the Planck fit of both lamps are shown in Figure 1.

\subsection{Experimental configuration}

Calibration experiments were conducted at the 'ShenguangII' laser facility platform. The optical path of the calibration is identical to the laser-induced shock experiments. Figure 2 shows a schematic of the calibration and verification configuration of the SOP system. A velocity interferometry system for any reflector (VISAR) is combined with the SOP system on the platform to permit simultaneous velocity and temperature measurements. The SOP system projects the emission from the standard lamp to a streak camera. An SC-10 streak camera produced by Optronis Company was used in our calibrations and experiments. The streak camera incorporates a streak tube with an S20 photocathode, a highvoltage power supply and control electronics. The dynamic sweep range of the camera is adjustable from $200 \mathrm{ps}$ to $100 \mathrm{~ms}$. The full-width at half-maximum (FWHM) of the line spread function (LSF) and the spectral response of the streak camera can be seen in Figures 3(a) and 3(b), 


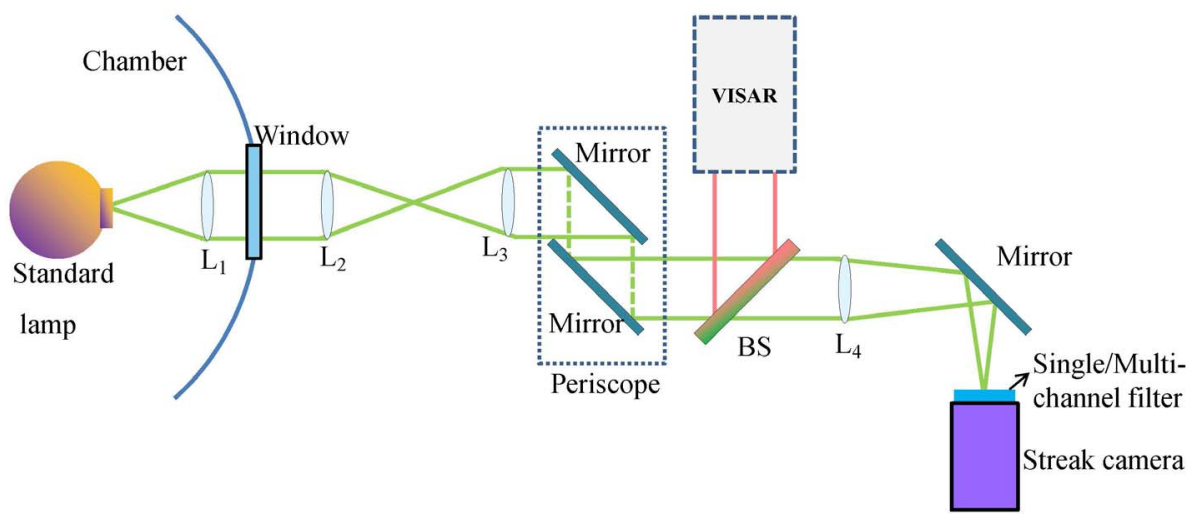

Figure 2. Calibration and verification configuration of the SOP system (top view).
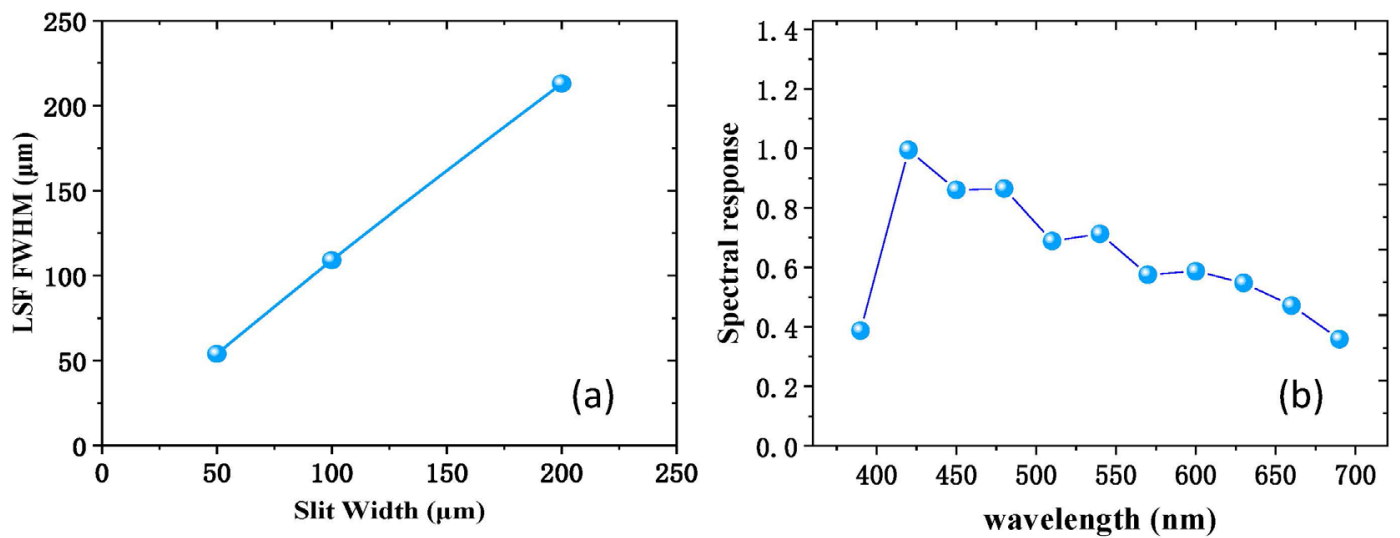

Figure 3. (a) LSF FWHM and (b) spectral response of the SC-10 streak camera.

respectively. The streak camera slit width used in our calibrations and experiments was $200 \mu \mathrm{m}$.

The outlet of the standard lamp is placed at the object plane of the imaging system. The first part of the imaging system (L1) is composed of several achromatic lenses to produce a magnified real image. This real image is transmitted and collimated by the compound lenses (L2 and L3). After passing through the periscope system, the projection is imaged by $\mathrm{L} 4$ at the slit of the SOP streak camera. The specially designed beam splitter (BS) is a dichroic mirror that reflects the probe beam $(660 \mathrm{~nm})$ to the VISAR while transmitting the thermal emission to the SOP. A singlechannel or multi-channel narrowband filter was placed in front of the slit of the streak camera to allow wavelengthspecific light transmission.

When starting the calibrations, the lower-color-temperature lamp (marked as Lamp A) was first placed on the object plane, as shown in Figure 2. Here, Lamp A is regarded as a known standard light source. Care was taken to ensure that the luminescent image of Lamp A was centered on the slit of the streak camera. A lens (L1) in the chamber was introduced to collect the emission for the same solid angle in different experiments and calibrations. After the light had passed through the SOP system, the emission was recorded on the streak camera during the luminous time. Calibrations were then carried out using microsecond sweeps ${ }^{[20]}$. The parameters related to the streak camera settings, such as the slits and micro-channel plate (MCP), must be the same in the calibrations to ensure consistency in the SOP transfer function. The streak camera was set to be in the dynamic mode, i.e., slow sweep mode, which is different from the static mode described by Miller et al. [5]. The camera output of a single pixel from the source is given by $C_{A}=t_{A} \eta_{A} \int_{\lambda} L_{A}(\lambda) \varphi_{A}(\lambda) \mathrm{d} \lambda$, where $t$ is the effective luminous time; $\eta$ is the count related to the solid angle, luminous area, gain of the camera, and slit width; $L(\lambda)$ is the spectral radiance; and $\varphi(\lambda)$ is the spectral response of the system. Next, the higher-color-temperature lamp (marked as Lamp B) was placed in the same position as Lamp A to ensure the same modulation transfer function between calibrations. Here, Lamp B is regarded as an unknown light source to be measured. The camera output of a single pixel from Lamp B is given by $C_{B}=t_{B} \eta_{B} \int_{\lambda} L_{B}(\lambda) \varphi_{B}(\lambda) \mathrm{d} \lambda$. Since the luminous modes of both lamps are similar and the slit width and camera gain were set to be the same in the calibrations, the relations $\varphi_{A}(\lambda)=\varphi_{B}(\lambda)$ and $\eta_{A}=\eta_{B}$ could be obtained. By dividing $C_{A}$ and $C_{B}$, we can obtain a simple relationship between both lamps, $L_{B}(\lambda)=L_{A}(\lambda)$. 


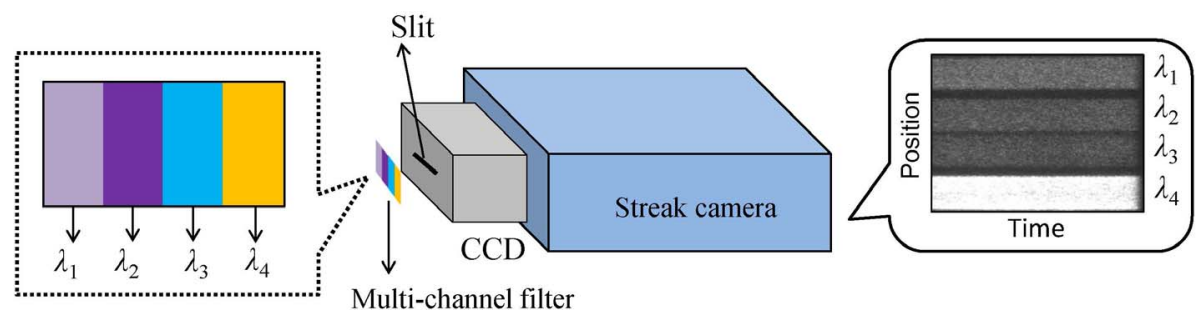

Figure 4. Schematic of the four-channel filter.

$\left(t_{A} / t_{B}\right) \cdot\left(C_{B} / C_{A}\right)$, assuming a small wavelength range of a single pixel ${ }^{[10]}$. Since $L_{A}(\lambda)$ is known and $t_{A}, t_{B}, C_{A}$ and $C_{B}$ can be obtained from the calibration experiments, $L_{B}(\lambda)$ can be calculated directly. A single-channel or multichannel narrowband filter was placed in front of the streak camera slit to determine the wavelength channel of the light transmission.

\subsection{Multi-channel method}

When the filter in front of the streak camera is a multichannel filter, the temperature of Lamp B can be obtained by fitting several $L_{B}(\lambda)$ values at different wavelengths $\lambda^{[12,13]}$. Here, a four-channel narrowband filter was adopted in the SOP system. A schematic of the filter is shown in Figure 4. Four different narrowband channels were successively and uniformly coated on a thin rectangular filter for $\lambda_{1}, \lambda_{2}, \lambda_{3}$ and $\lambda_{4}$, corresponding to $410,450,490$ and $590 \mathrm{~nm}$, respectively. The FWHM of each channel is less than $20 \mathrm{~nm}$ and the transmittance is greater than $80 \%$. As for the spectrometer such as iHR550, there will be large intensity loss after grating splitting by the spectrometer. Thus, the intensity of light at a specific wavelength will be reduced to $\sim 2 \%$ of the original intensity. Therefore, the advantage of the multichannel filter over a spectrometer is that the effective signal intensity is higher, making the signal-to-noise ratio better. The total width of the filter is the same as the horizontal width of the slit of the streak camera, while its height should be greater than that of the slit of the streak camera to cover the slit completely. Since the effective luminous time $t$ can be obtained from the streak camera, $t=s_{w} /$ pix, where $s_{w}$ is the sweep time of the streak camera and pix is the number of pixels on the entire screen. Using the equation $L_{B}(\lambda)=$ $L_{A}(\lambda) \cdot\left(t_{A} / t_{B}\right) \cdot\left(C_{B} / C_{A}\right)$, the spectral radiance of the lamp to be measured, $L_{B}(\lambda)$, can be obtained from the streak camera, and $L_{A}(\lambda)$ can be found by integrating the spectrum of each channel. The values of $t_{A}$ and $t_{B}$ are considered to be known. Given the calculated $L_{B}(\lambda)$ at different wavelengths, the emissivity and temperature can be simultaneously varied to obtain the best fit of the data using Equation (1), assuming that both parameters of Lamp B are unknown at this time. Here, the emissivity was considered as a constant or as linearly varying with wavelength. After fitting using the least squares method, values of $T_{B}=4893 \mathrm{~K}$ and $\varepsilon_{B}=$
$2.2 \times 10^{-4}$ were obtained, as shown in Figure 5(a). Since the original color temperature of Lamp B $\left(T_{B 0}\right)$ was $4956 \mathrm{~K}$ according to the original production test report, the relative deviation between $T_{B 0}$ and the measured $T_{B}$, expressed as $\eta_{B}=\left|T_{B}-T_{B 0}\right| / T_{B}=1.3 \%$ was obtained. The error of the spectral radiance has been analyzed according to Equation (9) in Section 3. The streak camera counts of calibrations of both lamps and the spectral radiance of a known lamp all contribute to the uncertainty.

Similarly, Lamp B was considered as the standard lamp source and Lamp A as the source to be measured. Then, four calculated $L_{A}(\lambda)$ values are given at different wavelengths. After fitting using the least squares method, values of $T_{A}=2904 \mathrm{~K}$ and $\varepsilon_{A}=0.007$ were obtained, as shown in Figure 5(b). The relative deviation gives $\eta_{A}=\left|T_{A}-T_{A 0}\right| / T_{A}=1.7 \%$. Details of the calibrations using the four-channel method are listed in Table 1. Also, the spatial chromaticity maps representing the measured temperature using Lamps A and B as the standard are shown in Figures 5(c) and 5(d), respectively. It can be seen from the chromaticity maps that the temperature obtained in each channel has a good spatial consistency. However, the temperatures obtained from different channels exhibit small differences. These differences are likely attributable to the variations in transmission spectra and camera responses of the different channels.

\subsection{Single-channel method}

If the filter in front of the streak camera is a single-channel filter, the temperature of Lamp B can be obtained from Equation (1) when using Lamp A as the standard. Here, $L_{B}(\lambda)$ can be directly determined from the formula $L_{B}(\lambda)=$ $L_{A}(\lambda) \cdot\left(t_{A} / t_{B}\right) \cdot\left(C_{B} / C_{A}\right)$, where $\lambda$ is the wavelength of the channel and the emissivity can be obtained from the original report of Lamp B, mentioned in Section 2.2. Similarly, the temperature of Lamp A can be measured when adopting Lamp B as the standard using the single-channel method. A series of calibration experiments were conducted at the 'Shenguang-II' laser facility platform, as summarized in Table 1. Therefore, the relative deviation $\eta$ reflects that the errors in temperature are less than $6 \%$ with the variation of wavelength in our calibrations, which indicates that the system is reliable at different wavelengths. 

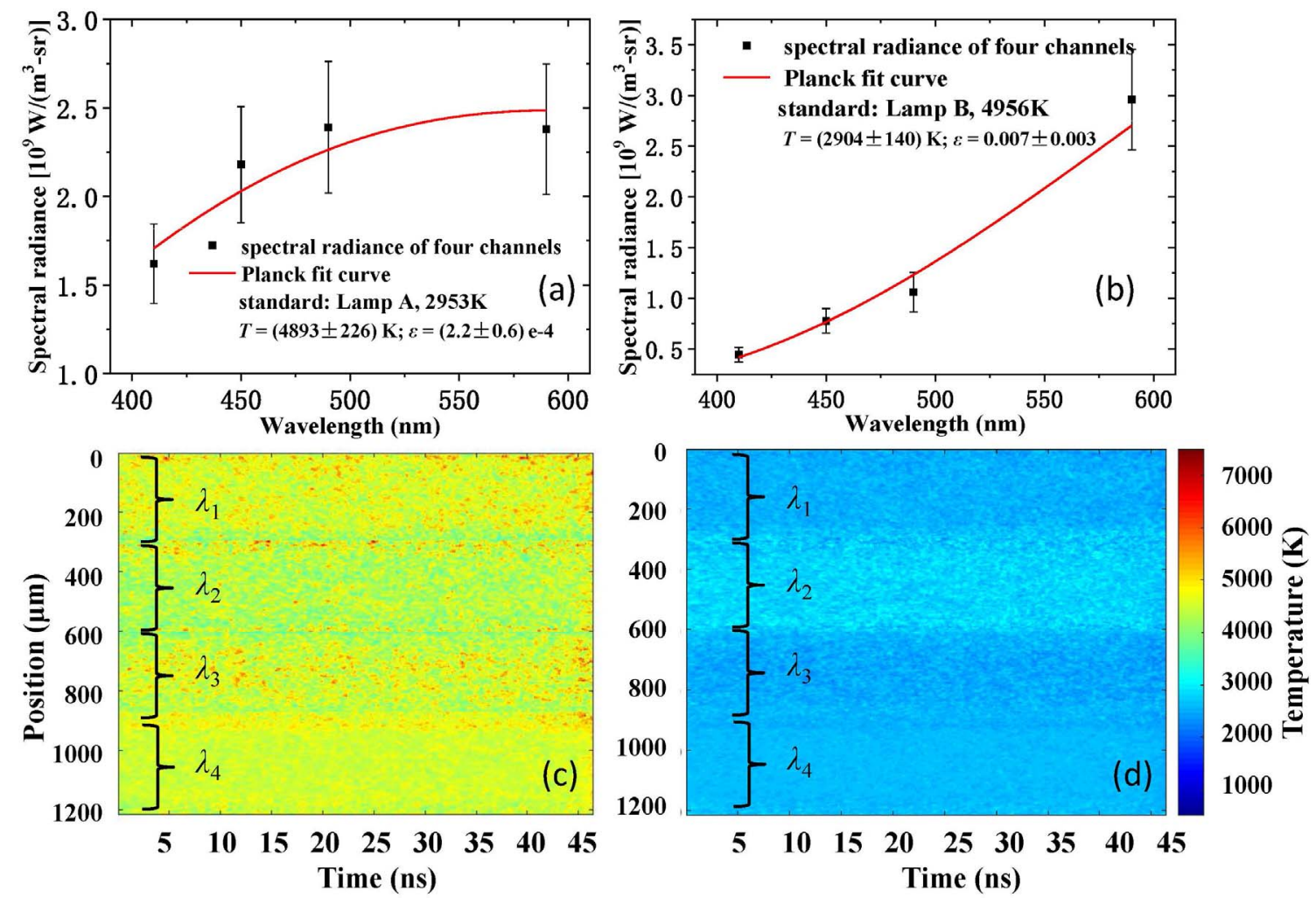

Figure 5. Spectral radiance data and theoretical Planck fit curve for conditions using (a) Lamp A as the standard or (b) Lamp B as the standard. Spatial chromaticity maps representing the measured temperature using (c) Lamp A as the standard or (d) Lamp B as the standard.

Table 1. Calibration and verification results (measured temperature $T$, deviation between the measured temperature and the standard value $\eta$ ) of the SOP system using a single-channel (No. S001-S0024) or multi-channel (No. M001-M002) method are listed in addition to the $\mathrm{MCP}$ of the streak camera for each experiment.

\begin{tabular}{|c|c|c|c|c|c|c|}
\hline \multirow[t]{2}{*}{ No. } & \multirow[t]{2}{*}{$\mathrm{MCP}$} & \multirow[t]{2}{*}{ Channel } & \multicolumn{2}{|c|}{ Standard: Lamp A } & \multicolumn{2}{|c|}{ Standard: Lamp B } \\
\hline & & & $T_{B}(\mathrm{~K})$ & $\eta_{B}(\%)$ & $T_{A}(\mathrm{~K})$ & $\eta_{A}(\%)$ \\
\hline$\overline{\mathrm{M} 001}$ & $850 \mathrm{~V}$ & Four-channel & 4893 & 1.3 & 2904 & 1.7 \\
\hline M002 & $800 \mathrm{~V}$ & Four-channel & 4870 & 1.8 & 2924 & 0.99 \\
\hline S001 & $750 \mathrm{~V}$ & Single-channel: $442 \mathrm{~nm}$ & 4801 & 3.2 & 2981 & 0.94 \\
\hline $\mathrm{S} 002$ & $800 \mathrm{~V}$ & Single-channel: $442 \mathrm{~nm}$ & 4818 & 2.9 & 2975 & 0.74 \\
\hline S003 & $850 \mathrm{~V}$ & Single-channel: $442 \mathrm{~nm}$ & 4839 & 2.4 & 2966 & 0.44 \\
\hline S0011 & $850 \mathrm{~V}$ & Single-channel: $410 \mathrm{~nm}$ & 4711 & 5.2 & 2981 & 0.94 \\
\hline S0012 & $850 \mathrm{~V}$ & Single-channel: $450 \mathrm{~nm}$ & 4844 & 2.3 & 2967 & 0.47 \\
\hline S0013 & $850 \mathrm{~V}$ & Single-channel: $490 \mathrm{~nm}$ & 4857 & 2.0 & 2978 & 0.84 \\
\hline S0014 & $850 \mathrm{~V}$ & Single-channel: $590 \mathrm{~nm}$ & 4749 & 4.3 & 3041 & 2.9 \\
\hline S0021 & $800 \mathrm{~V}$ & Single-channel: $410 \mathrm{~nm}$ & 4752 & 4.3 & 2990 & 1.2 \\
\hline S0022 & $800 \mathrm{~V}$ & Single-channel: $450 \mathrm{~nm}$ & 4879 & 1.6 & 2977 & 0.81 \\
\hline S0023 & $800 \mathrm{~V}$ & Single-channel: $490 \mathrm{~nm}$ & 4894 & 1.3 & 2983 & 1.0 \\
\hline S0024 & $800 \mathrm{~V}$ & Single-channel: $590 \mathrm{~nm}$ & 4782 & 3.6 & 3057 & 3.4 \\
\hline
\end{tabular}

\section{Uncertainty and sensitivity analysis of the SOP system}

For the multi-channel method, the uncertainty of the temperature and emissivity can be calculated by fitting the error from the least squares method ${ }^{[21]}$. The calculated uncertainties are listed in Table 2. For the single-channel method, the temperature $T$ can be obtained from Equation (1), and expressed as

$$
T=\frac{h c}{\lambda k \ln \left(\frac{2 h c^{2} \varepsilon}{L_{m} \lambda^{5}}+1\right)},
$$

where $L_{m}$ is the spectral radiance of the source to be measured. The emissivity $\varepsilon$ is assumed to be a constant in the calibrations and given by Kirchhoff's law as $\varepsilon=1-t-R$ in the shock-wave experiments ${ }^{[22]}$. The transmissivity $t$ is 
assumed to be zero since the shock-wave front is optically thick $^{[23]}$. The reflectivity of the shock front $R$ can be extracted from the VISAR data ${ }^{[24,25]}$ and was determined by comparing the shock reflectivity to that from the surface of the base material with a known value. Since the variables $\varepsilon$ and $L_{m}$ are measured by the VISAR and $\lambda$ is measured with a spectrophotometer, the three variables are measured independently. Then, the uncertainty in the temperature of the source to be measured can be expressed as

$\delta T=\sqrt{\left(\frac{\partial T}{\partial L_{m}}\right)^{2}\left(\delta L_{m}\right)^{2}+\left(\frac{\partial T}{\partial \lambda}\right)^{2}(\delta \lambda)^{2}+\left(\frac{\partial T}{\partial \varepsilon}\right)^{2}(\delta \varepsilon)^{2}}$,

according to the error-transfer rule ${ }^{[26,27]}$, where $\delta L_{m}, \delta \lambda$ and $\delta \varepsilon$ are the uncertainties of the spectral radiance, wavelength and emissivity, respectively. Because of the complexity of Equation (3), the components under the root sign in the formula have been calculated separately as

$$
\begin{aligned}
& \frac{\partial T}{\partial L_{m}}=\frac{h c}{\lambda k\left[\ln \left(\frac{2 h c^{2} \varepsilon}{L_{m} \lambda^{5}}+1\right)\right]^{2}\left(L_{m}+\frac{\lambda^{5}}{2 h c^{2} \varepsilon} L_{m}^{2}\right)} \\
& =\frac{T}{\ln \left(\frac{2 h c^{2} \varepsilon}{L_{m} \lambda^{5}}+1\right)\left(L_{m}+\frac{\lambda^{5}}{2 h c^{2} \varepsilon} L_{m}^{2}\right)}, \\
& \frac{\partial T}{\partial \lambda}=\frac{h c}{k}\left[\frac{5 \times 2 h c^{2} \varepsilon}{L_{m} \lambda^{7}\left[\ln \left(\frac{2 h c^{2} \varepsilon}{L_{m} \lambda^{5}}+1\right)\right]^{2}\left(\frac{2 h c^{2} \varepsilon}{L_{m} \lambda^{5}}+1\right)}\right. \\
& \left.-\frac{1}{\lambda^{2} \ln \left(\frac{2 h c^{2} \varepsilon}{L_{m} \lambda^{5}}+1\right)}\right] \\
& =T\left[\frac{5 \times 2 h c^{2} \varepsilon}{L_{m} \lambda^{6} \ln \left(\frac{2 h c^{2} \varepsilon}{L_{m} \lambda^{5}}+1\right)\left(\frac{2 h c^{2} \varepsilon}{L_{m} \lambda^{5}}+1\right)}-\frac{1}{\lambda}\right],
\end{aligned}
$$

$$
\begin{aligned}
\frac{\partial T}{\partial \varepsilon} & =-\frac{h c \times 2 h c^{2}}{\lambda^{6} k L_{m}\left[\ln \left(\frac{2 h c^{2} \varepsilon}{L_{m} \lambda^{5}}+1\right)\right]^{2}\left(\frac{2 h c^{2} \varepsilon}{L_{m} \lambda^{5}}+1\right)} \\
& =-T \frac{2 h c^{2}}{L_{m} \lambda^{5} \ln \left(\frac{2 h c^{2} \varepsilon}{L_{m} \lambda^{5}}+1\right)\left(\frac{2 h c^{2} \varepsilon}{L_{m} \lambda^{5}}+1\right)} .
\end{aligned}
$$

Substituting Equations (4)-(6) into Equation (3), $\delta T$ can be expressed as

$$
\begin{aligned}
\delta T= & \left\{\frac{\left(\delta L_{m}\right)^{2}}{\left[\ln \left(\frac{2 h c^{2} \varepsilon}{L_{m} \lambda^{5}}+1\right)\right]^{2}\left(L_{m}+\frac{\lambda^{5} L_{m}^{2}}{2 h c^{2} \varepsilon}\right)^{2}}\right. \\
& \times\left[\frac{5 \times 2 h c^{2} \varepsilon}{L_{m} \lambda^{6} \ln \left(\frac{2 h c^{2} \varepsilon}{L_{m} \lambda^{5}}+1\right)\left(\frac{2 h c^{2} \varepsilon}{L_{m} \lambda^{5}}+1\right)}-\frac{1}{\lambda}\right]^{2}(\delta \lambda)^{2} \\
+ & {\left.\left[\frac{2 h c^{2}(\delta \varepsilon)^{4}}{L_{m} \lambda^{5} \ln \left(\frac{2 h c^{2} \varepsilon}{L_{m} \lambda^{5}}+1\right)\left(\frac{2 h c^{2} \varepsilon}{L_{m} \lambda^{5}}+1\right)}\right]^{2}\right]^{1 / 2} . }
\end{aligned}
$$

The relative uncertainty of $T$ can be expressed as

$$
\begin{aligned}
& \frac{\delta T}{T}=\left\{\frac{\left(\delta L_{m}\right)^{2}}{\left[\ln \left(\frac{2 h c^{2} \varepsilon}{L_{m} \lambda^{5}}+1\right)\right]^{2}\left(L_{m}+\frac{\lambda^{5} L_{m}^{2}}{2 h c^{2} \varepsilon}\right)^{2}}\right]^{2} \\
&+\left[\frac{5 \times 2 h c^{2} \varepsilon}{L_{m} \lambda^{6} \ln \left(\frac{2 h c^{2} \varepsilon}{L_{m} \lambda^{5}}+1\right)\left(\frac{2 h c^{2} \varepsilon}{L_{m} \lambda^{5}}+1\right)}-\frac{1}{\lambda}\right]^{2}(\delta \lambda)^{2} \\
&+\left[\frac{2 h c^{2}(\delta \varepsilon)^{4}}{L_{m} \lambda^{5} \ln \left(\frac{2 h c^{2} \varepsilon}{L_{m} \lambda^{5}}+1\right)\left(\frac{2 h c^{2} \varepsilon}{L_{m} \lambda^{5}}+1\right)}\right]^{1 / 2}
\end{aligned}
$$

Thus, to obtain $\delta T$, the components $\delta L_{m}, \delta \lambda$, and $\delta \varepsilon$ need to be calculated independently.

The values of $\delta L_{m}$ can be obtained from $L_{m}=L_{s}$. $\left(t_{s} / t_{m}\right) \cdot\left(C_{m} / C_{s}\right)$, as mentioned in Section 2.2, according to the error-transfer formula, where the subscripts $m$ and $s$ represent the source to be measured and standard source, respectively. Since $t_{s}$ and $t_{m}$ are constants related to the streak camera, the uncertainty in the spectral radiance can be expressed as

$$
\begin{gathered}
\delta L_{m}=\sqrt{\left(\frac{\partial L_{m}}{\partial C_{m}}\right)^{2}\left(\delta C_{m}\right)^{2}+\left(\frac{\partial L_{m}}{\partial C_{s}}\right)^{2}\left(\delta C_{s}\right)^{2}+\left(\frac{\partial L_{m}}{\partial L_{s}}\right)^{2}\left(\delta L_{s}\right)^{2}} \\
=L_{m} \sqrt{\left(\frac{\delta C_{m}}{C_{m}}\right)^{2}+\left(\frac{\delta C_{s}}{C_{s}}\right)^{2}+\left(\frac{\delta L_{s}}{L_{s}}\right)^{2}}
\end{gathered}
$$

where $\delta C / C$ is $\sim 10 \%$ according to the standard deviation of the streak camera, and $\delta L_{S} / L_{S}$ is $\sim 5 \%$ according to 
the calibration report of the standard lamps. Therefore, the relative uncertainty of the spectral radiance $\delta L_{m} / L_{m}$ is $\sim 15 \%$.

The values of $\delta \lambda$ can be measured by the spectrophotometer, which manifests as the maximum deviation from the central wavelength. Then, the relative uncertainty $\delta \lambda / \lambda$ can be obtained as well. For filters with a large bandwidth, the error is also potentially significant.

In calibration experiments, $\varepsilon, \delta \varepsilon$ and $\delta \varepsilon / \varepsilon$ can be obtained directly from the original calibration report of the standard lamps. However, in shock-wave experiments, $\varepsilon=1-$ $R_{\mathrm{SOP}}$, which gives $\delta \varepsilon=\delta R_{\mathrm{SOP}}$. Since $R_{\mathrm{SOP}}=R_{\mathrm{VISAR}}-$ $d=R_{\text {base }} C_{\mathrm{VISAR}} / C_{\text {base }}-d$, where $d$ is the difference in reflectivity between the SOP channel and the VISAR probe beam, it can be regarded as a constant in a certain state ${ }^{[10,11]}$. $R_{\text {base }}$ is the reflectivity of base material, while $C_{\mathrm{VISAR}}$ and $C_{\text {base }}$ are the camera counts of the shocked material and base material, respectively. The uncertainty of the emissivity in the shock-wave experiments can be expressed as

$$
\begin{aligned}
\delta \varepsilon & =\delta R_{\mathrm{SOP}}=\delta R_{\mathrm{VISAR}} \\
& =R_{\mathrm{VISAR}} \sqrt{\left(\frac{\delta C_{\mathrm{VISAR}}}{C_{\mathrm{VISAR}}}\right)^{2}+\left(\frac{\delta C_{\text {base }}}{C_{\text {base }}}\right)^{2}+\left(\frac{\delta R_{\text {base }}}{R_{\text {base }}}\right)^{2}},
\end{aligned}
$$

where $\delta C_{\mathrm{VISAR}} / C_{\mathrm{VISAR}} \approx \delta C_{\text {base }} / C_{\text {base }}$ is $\sim 10 \%$, and $R_{\text {base }}, \delta R_{\text {base }}$, and $\delta R_{\text {base }} / R_{\text {base }}$ should be measured by the target group.

Therefore, the uncertainty and relative uncertainty of the temperature to be measured, $\delta T$ and $\delta T / T$, can be calculated by substituting $\delta L_{m}, \delta \lambda$ and $\delta \varepsilon$ into Equations (7) and (8). The calculated uncertainties in the calibrations and shockwave experiments are listed in Table 2.

The precision of the temperature calculation can be improved by analyzing its sensitivity to the variables $L_{m}, \lambda$ and $\varepsilon$. The sensitivity $S$ can be defined as

$$
S_{X}=\frac{\Delta T / T}{\Delta X / X}
$$

where $X$ refers to three variables that affect the temperature calculation. Figure 6(a) shows the relationship between $\Delta T / T$ and $\Delta X / X$, while Figure 6(b) shows the temperature sensitivity of the three variables in a single calibration experiment. The results show that the wavelength $\lambda$ is the most sensitive to the temperature calculation within the vicinity of the measured value of thousands of kelvin. The temperature sensitivities of the emissivity and radiation intensity are smaller than that of the wavelength, and are similar to each other, but in opposite directions.

For the shock-wave experiments shown in Figures 6(c) and 6(d), all of the variables are far more sensitive to the temperature than during the calibration, leading to greater errors in the temperature calculation. The wavelength is the most sensitive variable to the temperature in the shock-wave experiments but with a direction opposite to that during the calibration. The sensitivities of the two other variables are similar in the vicinity of the measured value, but in opposite directions as well. Although the wavelength seems to be the most sensitive factor, its uncertainty is the smallest of the three variables in the shock-wave experiments according to Table 2. Concretely, for the shock-wave experiment D0211, $\delta \lambda / \lambda \sim 2.2 \%$, where $S_{\lambda} \sim 2.6$ and $\Delta T / T \sim 5.7 \%$. Also, $\delta \varepsilon / \varepsilon \sim 15 \%$, where $S_{\varepsilon} \sim-0.8$ and $\Delta T / T \sim-10 \%$ and $\delta L_{m} / L_{m} \sim 15 \%$, where $S_{L} \sim 0.75$ and $\Delta T / T \sim 9.7 \%$. In general, even if $\lambda$ is the most sensitive variable, $\varepsilon$ and $L_{m}$ have greater influence on the temperature calculation. The uncertainty of $L_{m}$ originates from the performance of the streak camera, while the uncertainty of $\varepsilon$ mainly arises from the reflectivity measurement. Thus, improving the precision of the reflectivity measurement is the key to improving the precision of the temperature calculation.

\section{Applications in shock experiments}

To verify the reliability of the SOP system for temperature measurements at tens of thousands of kelvin, quartz was selected as the material to be studied, owing to its abundant research data. A series of laser-induced shock experiments were conducted at the 'Shenguang-II' laser facility. Decaying shock waves were generated by ablation of the thin plastic layers $(\mathrm{CH}$ and $\mathrm{CHBr})$ backing the sample. Laser energies of up to $1000 \mathrm{~J}$ were delivered at $351 \mathrm{~nm}$. The temporal profile of the laser was nearly square, with an FWHM of $\sim 2$ ns. A lens-array (LA) system ${ }^{[28,29]}$ was used to smooth the laser beam to obtain a flat-topped profile in the focal plane. The focal spot had a $1 \mathrm{~mm} \times 0.7 \mathrm{~mm}$ rectangular region, and the resulting intensities were $\sim 10^{14} \mathrm{~W} / \mathrm{cm}^{2}$.

Standard laser-shock diagnostics, including SOP and VISAR, were employed. The VISAR $(660 \mathrm{~nm})$ was applied for simultaneous diagnostics with the SOP for the free surface velocity and optical reflectivity. The diagnostic systems had a temporal resolution of $\sim 50 \mathrm{ps}$ and a spatial resolution of $\sim 7 \mu \mathrm{m}$.

Figures 7(a)-7(c) show the target and diagnostic images of one of the shock experiments. The shock velocity, reflectivity and self-emission of the sample were obtained from the VISAR and SOP system. Figures 7(d) and 7(e) show the reflectivity and the temperature as functions of the shock velocity in both quartz and fused silica. The velocities were measured to $\sim 1 \%$ precision in our experiments ${ }^{[8]}$. Our reflectivity curve of quartz was almost coincident with Hicks' data ${ }^{[2]}$, except for the smaller data around $18 \mathrm{~km} / \mathrm{s}$. As for the reflectivity of fused silica, our data below $18 \mathrm{~km} / \mathrm{s}$ seemed to agree with Hicks' data. But the saturated reflectivity was $\sim 25 \%$ higher than Hicks' data, which might be due to differences in materials such as density and surface 
Table 2. Variables and their uncertainties in the calibrations and shock-wave experiments. Samples for the calibrations using a singlechannel begin with an S; samples for calibrations using a multi-channel begin with an $\mathrm{M}$; and samples for the shock-wave experiments begin with a $\mathrm{D}$. In particular, the $L_{m}, \varepsilon$, and $T$ of the shock-wave experiments refer to values at the interface of the sample and base material.

\begin{tabular}{|c|c|c|c|c|c|c|c|c|c|}
\hline No. & Sample & $\begin{array}{c}L_{m} \\
\left(\mathrm{~W} /\left(\mathrm{m}^{3} \cdot \mathrm{sr}\right)\right)\end{array}$ & $\begin{array}{c}\delta L_{m} \\
\left(\mathrm{~W} /\left(\mathrm{m}^{3} \cdot \mathrm{sr}\right)\right)\end{array}$ & $\begin{array}{c}\lambda \\
(\mathrm{nm})\end{array}$ & $\begin{array}{c}\delta \lambda \\
(\mathrm{nm})\end{array}$ & $\varepsilon$ & $\delta \varepsilon$ & $\begin{array}{c}T \\
(\mathrm{~K})\end{array}$ & $\begin{array}{l}\delta T \\
(\mathrm{~K})\end{array}$ \\
\hline M001B & Lamp B & & Four-channel & & & $2.2 \times 10^{-4}$ & $6.0 \times 10^{-5}$ & 4893 & 226 \\
\hline M002B & Lamp B & & Four-channel & & & $2.3 \times 10^{-4}$ & $9.8 \times 10^{-5}$ & 4870 & 217 \\
\hline S001B & Lamp B & $1.99 \times 10^{9}$ & $2.99 \times 10^{8}$ & 442 & 20 & $2.5 \times 10^{-4}$ & $2.7 \times 10^{-6}$ & 4799 & 121 \\
\hline S002B & Lamp B & $2.04 \times 10^{9}$ & $3.06 \times 10^{8}$ & 442 & 20 & $2.5 \times 10^{-4}$ & $2.7 \times 10^{-6}$ & 4817 & 121 \\
\hline S003B & Lamp B & $2.10 \times 10^{9}$ & $3.15 \times 10^{8}$ & 442 & 20 & $2.5 \times 10^{-4}$ & $2.7 \times 10^{-6}$ & 4838 & 122 \\
\hline S0011B & Lamp B & $1.49 \times 10^{9}$ & $2.23 \times 10^{8}$ & 410 & 10 & $2.5 \times 10^{-4}$ & $2.7 \times 10^{-6}$ & 4711 & 102 \\
\hline S0012B & Lamp B & $2.18 \times 10^{9}$ & $3.27 \times 10^{8}$ & 450 & 10 & $2.5 \times 10^{-4}$ & $2.7 \times 10^{-6}$ & 4843 & 113 \\
\hline S0013B & Lamp B & $2.48 \times 10^{9}$ & $3.72 \times 10^{8}$ & 490 & 15 & $2.5 \times 10^{-4}$ & $2.7 \times 10^{-6}$ & 4854 & 123 \\
\hline S0014B & Lamp B & $2.45 \times 10^{9}$ & $3.68 \times 10^{8}$ & 590 & 15 & $2.5 \times 10^{-4}$ & $2.7 \times 10^{-6}$ & 4747 & 138 \\
\hline S0021B & Lamp B & $1.52 \times 10^{9}$ & $2.28 \times 10^{8}$ & 410 & 10 & $2.5 \times 10^{-4}$ & $2.7 \times 10^{-6}$ & 4725 & 103 \\
\hline S0022B & Lamp B & $2.26 \times 10^{9}$ & $3.39 \times 10^{8}$ & 450 & 10 & $2.5 \times 10^{-4}$ & $2.7 \times 10^{-6}$ & 4869 & 114 \\
\hline S0023B & Lamp B & $2.56 \times 10^{9}$ & $3.84 \times 10^{8}$ & 490 & 15 & $2.5 \times 10^{-4}$ & $2.7 \times 10^{-6}$ & 4880 & 124 \\
\hline S0024B & Lamp B & $2.49 \times 10^{9}$ & $3.73 \times 10^{8}$ & 590 & 15 & $2.5 \times 10^{-4}$ & $2.7 \times 10^{-6}$ & 4762 & 139 \\
\hline M001A & Lamp A & & Four-channel & & & $7.0 \times 10^{-3}$ & $3.0 \times 10^{-3}$ & 2904 & 140 \\
\hline M002A & Lamp A & & Four-channel & & & $7.3 \times 10^{-3}$ & $2.5 \times 10^{-3}$ & 2924 & 50 \\
\hline S001A & Lamp A & $7.68 \times 10^{8}$ & $1.15 \times 10^{8}$ & 442 & 20 & $6.1 \times 10^{-3}$ & $3.9 \times 10^{-5}$ & 2981 & 84 \\
\hline S002A & Lamp A & $7.52 \times 10^{8}$ & $1.13 \times 10^{8}$ & 442 & 20 & $6.1 \times 10^{-3}$ & $3.9 \times 10^{-5}$ & 2975 & 84 \\
\hline S003A & Lamp A & $7.29 \times 10^{8}$ & $1.09 \times 10^{8}$ & 442 & 20 & $6.1 \times 10^{-3}$ & $3.9 \times 10^{-5}$ & 2967 & 84 \\
\hline S0011A & Lamp A & $4.78 \times 10^{8}$ & $7.17 \times 10^{7}$ & 410 & 10 & $6.1 \times 10^{-3}$ & $3.9 \times 10^{-5}$ & 2982 & 56 \\
\hline S0012A & Lamp A & $8.12 \times 10^{8}$ & $1.22 \times 10^{8}$ & 450 & 10 & $6.1 \times 10^{-3}$ & $3.9 \times 10^{-5}$ & 2968 & 54 \\
\hline S0013A & Lamp A & $1.32 \times 10^{9}$ & $1.98 \times 10^{8}$ & 490 & 15 & $6.1 \times 10^{-3}$ & $3.9 \times 10^{-5}$ & 2977 & 64 \\
\hline S0014A & Lamp A & $3.30 \times 10^{9}$ & $4.95 \times 10^{8}$ & 590 & 15 & $6.1 \times 10^{-3}$ & $3.9 \times 10^{-5}$ & 3040 & 64 \\
\hline S0021A & Lamp A & $4.81 \times 10^{8}$ & $7.21 \times 10^{7}$ & 410 & 10 & $6.1 \times 10^{-3}$ & $3.9 \times 10^{-5}$ & 2983 & 57 \\
\hline S0022A & Lamp A & $8.38 \times 10^{8}$ & $1.26 \times 10^{8}$ & 450 & 10 & $6.1 \times 10^{-3}$ & $3.9 \times 10^{-5}$ & 2976 & 54 \\
\hline S0023A & Lamp A & $1.36 \times 10^{9}$ & $2.04 \times 10^{8}$ & 490 & 15 & $6.1 \times 10^{-3}$ & $3.9 \times 10^{-5}$ & 2986 & 64 \\
\hline S0024A & Lamp A & $3.50 \times 10^{9}$ & $5.25 \times 10^{8}$ & 590 & 15 & $6.1 \times 10^{-3}$ & $3.9 \times 10^{-5}$ & 3062 & 65 \\
\hline D0211 & Quartz & $4.61 \times 10^{15}$ & $6.91 \times 10^{14}$ & 450 & 10 & 0.707 & 0.109 & 46498 & 7727 \\
\hline D0212 & Quartz & $1.80 \times 10^{15}$ & $2.70 \times 10^{14}$ & 590 & 15 & 0.720 & 0.111 & 47710 & 8787 \\
\hline D0213 & Fused silica & $4.37 \times 10^{15}$ & $6.55 \times 10^{14}$ & 410 & 10 & 0.534 & 0.082 & 43134 & 6847 \\
\hline D0214 & Fused silica & $3.65 \times 10^{15}$ & $5.47 \times 10^{14}$ & 490 & 15 & 0.534 & 0.082 & 61117 & 11836 \\
\hline
\end{tabular}

roughness. The size of the uncertainty in the calculated temperature was $15 \%-18 \%$ according to Section 3. Compared with previous experiments, the temperature of the quartz here was nearly consistent with Hicks' data, but slightly lower at shock velocities below $20 \mathrm{~km} / \mathrm{s}$. In fact, since the acquired reflectivity was continuous data in one shot, the error of reflectivity measured by VISAR intensity would become gradually larger with the attenuation of the shock wave and the influence of the lateral release wave. This is probably the reason for the lower temperature of quartz around $18 \mathrm{~km} / \mathrm{s}$ in our experiments. Analysis in Section 3 does not consider the influence of the release wave on reflectivity intensity, which will also lead to differences of the final temperature results. In the future, there is still space and necessity to study the error of the gray body hypothesis itself and the influence of the release wave on reflectivity. Also, the temperature of fused silica was $\sim 25 \%$ higher than that of quartz. This observation is also consistent with Hicks' results. However, both experimental data on the laser are $\sim 28 \%$ lower than in the Sesame model ${ }^{[30]}$. Therefore, the measured temperatures of the standard materials in our experiments agreed fairly well with those obtained in previous works, which could be evidence of the reliability of the SOP system at tens of thousands of kelvin.

\section{Conclusions}

Two calibrated Planckian radiators with different color temperatures were used for calibration and verification of the SOP system. They are regarded as both the known standard and unknown source for each other in the SOP system. The SOP system was calibrated using both multi-channel and single-channel methods. To verify the reliability of the SOP system, the relative deviation between the measured data and the standard value was calibrated for. Also, a method to analyze the uncertainty and sensitivity of the SOP system was proposed. To verify the reliability of the SOP system for 

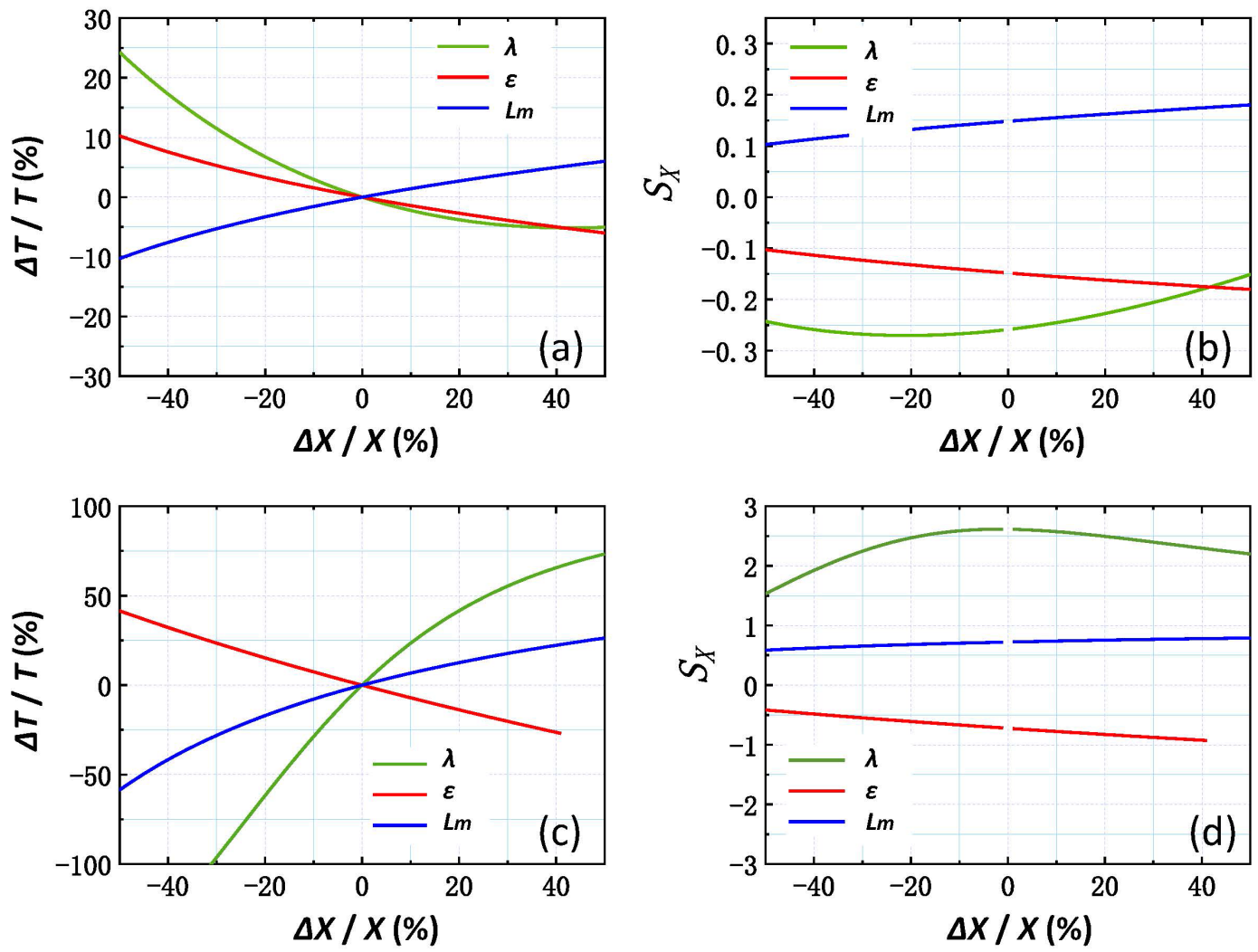

Figure 6. Relationship between $\Delta T / T$ and $\Delta X / X$ and the sensitivity curve of the calibration (No. S003B, (a) and (b)) and shock-wave experiment (No. D0211, (c) and (d)).
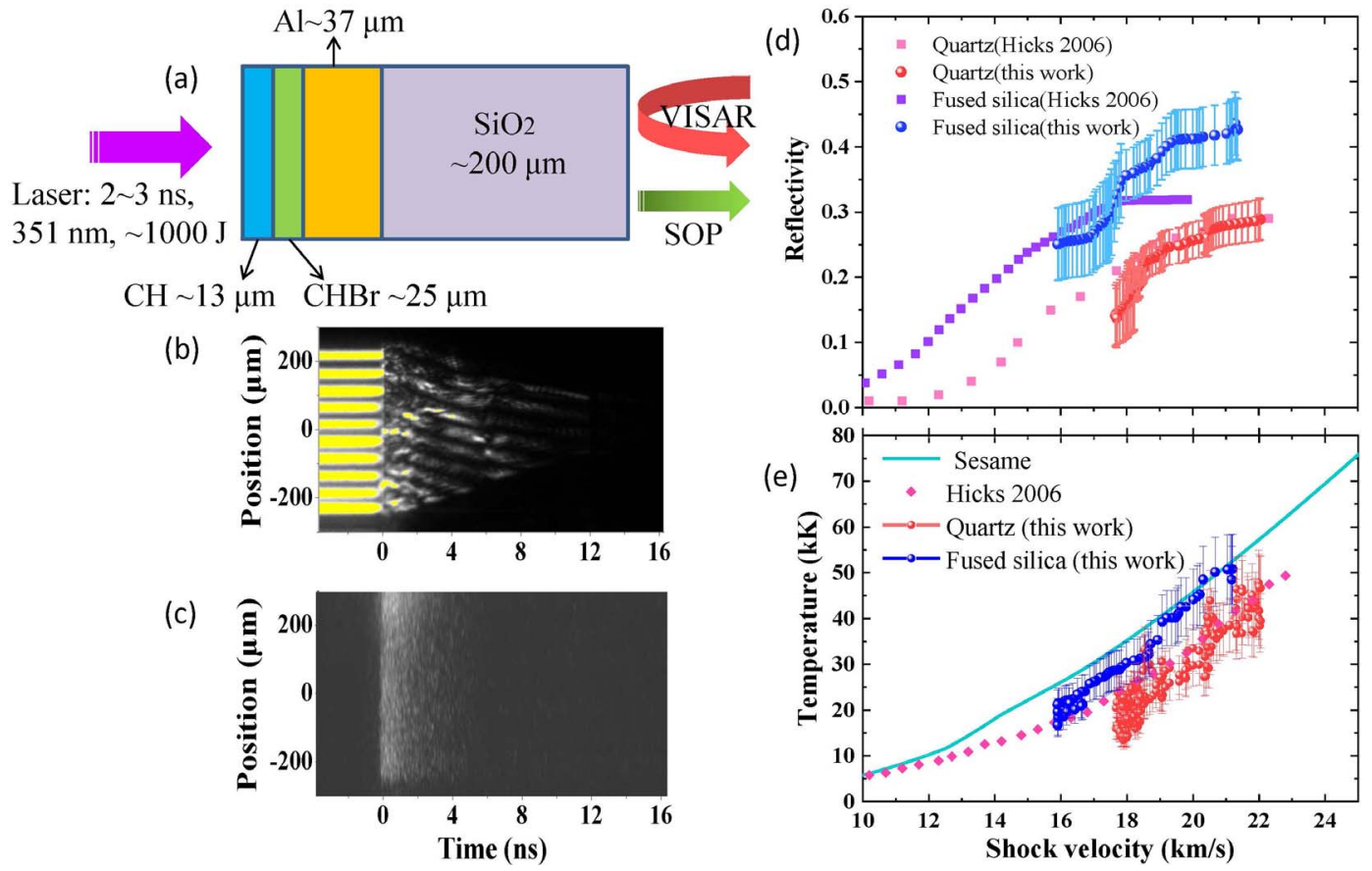

Figure 7. (a) Schematic of the target; (b) VISAR line-image record; (c) SOP image record; (d) a comparison of the reflectivity as a function of shock velocity in quartz and fused silica by Hicks et al. ${ }^{[2,6]}$; and (e) a comparison of the measured temperature as a function of shock velocity in quartz by Hicks et al. (solid pink diamonds), the Sesame model (solid cyan line), and this work (solid blue circles for fused silica and solid red circles for quartz). 
temperature measurements at tens of thousands of kelvin, a series of laser-induced shock experiments were conducted at the 'Shenguang-II' laser facility. The measured temperature of quartz in our experiments agreed with previous works fairly well, which could be evidence of the reliability of the SOP system.

\section{Acknowledgements}

We gratefully acknowledge the valuable support for the experiments by the 'Shenguang-II' technical crews. This work was supported by the National Key R\&D Program of China (No. 2017YFA0403200) and the Science Challenge Project (No. TZ2016001).

\section{References}

1. R. P. Drake, High Energy Density Physics (Springer, New York, 2006).

2. D. G. Hicks, T. R. Boehly, J. H. Eggert, J. E. Miller, P. M. Celliers, and G. W. Collins, Phys. Rev. Lett. 97, 025502 (2006).

3. Ya. B. Zeldovich and Yu. P Raizer, Physics of Shock Waves and High-Temperature Hydrodynamic Phenomena (Academic Press, New York, 1966), p. 86.

4. J. R. Asay, M. Shahinpoor, and L. Davison, High-Pressure Shock Compression of Solids (Springer, New York, 1993).

5. J. E. Miller, T. R. Boehly, A. Melchior, D. D. Meyerhofer, P. M. Celliers, J. H. Eggert, D. G. Hicks, C. M. Sorce, J. A. Oertel, and P. M. Emmel, Rev. Sci. Instrum. 78, 034903 (2007).

6. D. G. Hicks, T. R. Boehly, P. M. Celliers, J. H. Eggert, E. Vianello, D. D. Meyerhofer, and G. W. Collins, Phys. Plasmas 12, 082702 (2005).

7. T. Sano, N. Ozaki, T. Sakaiya, K. Shigemori, M. Ikoma, T. Kimura, K. Miyanishi, T. Endo, A. Shiroshita, and H. Takahashi, Phys. Rev. B 83, 3002 (2011).

8. H. Shu, S. Z. Fu, X. G. Huang, Z. H. Fang, T. Wang, J. J. Ye, Z. Y. Xie, H. Z. Zhou, and T. Long, Eur. Phys. J. D 66, 268 (2012).

9. H. Shu, S. Fu, X. Huang, J. Wu, Z. Xie, F. Zhang, J. Ye, G. Jia, and H. Zhou, Phys. Plasmas 21, 2162 (2014).

10. Z. He, G. Jia, F. Zhang, K. Luo, X. Huang, H. Shu, Z. Fang, J. Ye, Z. Xie, M. Xia, and S. Fu, Eur. Phys. J. D 72, 3 (2018).
11. Z. He, H. Shu, X. Huang, Q. Zhang, G. Jia, F. Zhang, Y. Tu, J. Wang, J. Ye, Z. Xie, Z. Fang, W. Pei, and S. Fu, Chin. Phys. B 27, 8 (2018).

12. G. A. Lyzenga and T. J. Ahrens, Rev. Sci. Instrum. 50, 1421 (1979).

13. Z. He, H. Zhou, X. Huang, G. Jia, H. Shu, Z. Fang, J. Ye, and Z. Xie, High Power Laser Part. Beams 28, 042002 (2016).

14. T. Morita, Y. Kuramitsu, H. Tanji, H. Aoki, T. Ide, S. Shibata, N. Onishi, C. Gregory, A. Diziere, and J. Waugh, Astrophys. Space Sci. 336, 283 (2011).

15. T. Morita, Y. Sakawa, Y. Kuramitsu, S. Dono, T. Ide, S. Shibata, H. Aoki, H. Tanji, T. Sano, and A. Shiroshita, Rev. Sci. Instrum. 83, 10D514 (2012).

16. M. C. Gregor, R. Boni, A. Sorce, J. Kendrick, C. A. Mccoy, D. N. Polsin, T. R. Boehly, P. M. Celliers, G. W. Collins, and D. E. Fratanduono, Rev. Sci. Instrum. 87, 114903 (2016).

17. T. Hua, Introduction to Experimental Shock-Wave Physics (National Defense Industry Press, Beijing, 2007).

18. D. P. Higginson, J. M. Mcnaney, D. C. Swift, T. Bartal, D. S. Hey, R. Kodama, S. Pape, A. Le Mackinnon, D. Mariscal, and H. Nakamura, Phys. Plasmas 17, 1003 (2010).

19. J. C. Fernandez, C. W. Barnes, M. J. Mocko, and L. Zavorka, Sensitivity Analysis and Requirements for Temporally and Spatially Resolved Thermometry Using Neutron Resonance Spectroscopy, LANL Report LA-UR-18-20686 (2018).

20. D. K. Spaulding, Laser-Driven Shock Compression Studies of Planetary Compositions (University of California, Berkeley, 2010).

21. X. Pang, Mathematical Analysis (Higher Education Press, Shanghai, 2010).

22. V. D. Glukhodedov and S. I. Kirshanov, J. Exp. Theor. Phys. 89, 292 (1999).

23. S. B. Kormer, Sov. Phys. Usp. 11, 229 (1968).

24. P. M. Celliers, D. K. Bradley, G. W. Collins, D. G. Hicks, T. R. Boehly, and W. J. Armstrong, Rev. Sci. Instrum. 75, 4916 (2004).

25. X. Zhou, W. J. Nellis, J. Li, J. Li, W. Zhao, X. Liu, X. Cao, Q. Liu, T. Xue, and Q. Wu, J. Appl. Phys. 118, 043524 (2015).

26. S. Fu, X. Huang, M. Ma, H. Shu, J. Wu, J. Ye, J. He, Y. Gu, P. Luo, and T. Rong, J. Appl. Phys. 101, 043517 (2007).

27. P. M. Celliers, G. W. Collins, D. G. Hicks, and J. H. Eggert, J. Appl. Phys. 98, 113529 (2005).

28. X. Deng, X. Liang, Z. Chen, W. Yu, and R. Ma, Appl. Opt. 25, 377 (1986).

29. S. Fu, Y. Gu, J. Wu, and S. Wang, Phys. Plasmas 2, 3461 (1995).

30. S. P. Lyon and J. D. Johnson, SASAME: The Los Alamos National Laboratory Equation of State Database, LANL Report LA-UR-92-3407 (1992). 